\title{
Thermal residual stresses in particulate composites and its toughening effect
}

\author{
Zhong Ling · Yong-Li Wu
}

Received: 25 March 2004 / Accepted: 13 April 2006/ Published online: 19 January 2007

(C) Springer Science+Business Media, LLC 2007

\begin{abstract}
In this paper, an accurate formula for calculating the thermal residual stress field in a particle-reinforced composite are presented. Numerical examples are given to show r-variations of the thermal residual stresses. The increase in fracture toughness of matrix predicted by the thermal residual stress field is compared well with the experimentally measured increase.
\end{abstract}

\section{Introduction}

It has been recognized for some time that the dispersion of second-phase particles can increase the fracture behavior of ceramics. Whenever a multiphase microstructure experiences hot-pressing at elevated temperature, the mismatch between the coefficients of the thermal expansion and elastic constants in matrix and particle results in generation of residual stresses in particle and matrix upon cooling to room temperature. These residual thermo-elastic micromechanical stresses have always been of interest from both the strengthening and the weakening perspectives.

Selsing [1] presented a solution for the thermal residual stress of a single spherical inclusion imbedded within an infinite isotropic elastic matrix, the two

This work was supported by the National Natural Science Foundation of China (No.50172053).

Z. Ling · Y.-L. Wu ( $ه)$

State Key LNM, Institute of Mechanics, CAS, 100080 Beijing, China

e-mail: wuyL0531@yahoo.com.cn having different coefficient of thermal expansion and elastic constants.

$\sigma_{r m}=\sigma_{h}=\frac{\left(\alpha_{p}-\alpha_{m}\right)}{\left(1+v_{m}\right) / 2 E_{m}+\left(1-2 v_{p}\right) / E_{p}}$

where $\alpha, E, v$ are the coefficient of thermal expansion, elastic modulus and Poisson's ratio, the subscripts $m$ and $p$ refer to the matrix and the particular respectively, $\Delta T=T_{R}-T_{P}, T_{R}$ and $T_{P}$ denote the room temperature and the processing temperature. Wei and Becher [2] consider the thermal residual stress (1) which is induced by CTE mismatch as a major cause for the crack deflection. The Eq. (1) does not contain the volume fraction of particle $f_{p}$. It is a lowerorder approximation to the stress in composite with a finite volume fraction of particle. Although they made no quantitative estimate of the increased toughness.

Another toughening mechanism is the thermal residual stresses in a particulate composite [3-5]. Virkar and Johnson [3] showed that micromechanical residual stresses can enhance the fracture toughness of ceramics-metal composites. However, the periodic nature of the residual stresses was not fully incorporated in the analysis in that internal stresses acting on only half the wavelength were considered. Culter and Virkar [4] postulated a periodic tension-compression residual stresses field, which was caused by CTE mismatch as the composite was cooled to room temperature, in a $\mathrm{ZrO}_{2}-\mathrm{Zr}$ composite. This periodic residual stress model was used to explain the toughness increase which was observed in the $\mathrm{ZrO}_{2}-\mathrm{Zr}$ composite. The model proposed by Evans et al. [5] and Cutler and Virkar [4] provided the fracture toughness, $K_{I C}$, of a particulate due to periodic residual stress field as 
$K_{I C}=K_{I 0}+2 q \sqrt{2 D / \pi}$

where $K_{I 0}$ is the critical stress intensity factor of the matrix, $q$ is the local residual compressive stress, and $D$ is the length of the compressive stress zone which in this case is the average particulate spacing.

Equation (2) is based on a stress intensity factor solution by Tada et al. [6] for a semi-infinite twodimensional crack with a compressive stress zone of intensity and length $D$. The compressive thermal residual stress in matrix is generated when the CTE of the particle exceeds that of the matrix. It was found [4] that $q$ is a strong function of the volume fraction of second-phase particles and large values of $q$ resulted in large $K_{I C}$.

Taya et al. [7] presented a formula for calculating the average residual stresses in the particle and the matrix by using the modified Eshelby model [8] which consider a finite volume fraction of particles.

In this paper, an accurate formula for calculating the residual stresses in the particle and the matrix of the particle composite will be presented. The results show that the residual stresses distribution in the matrix outer the particle are not uniform which considered by Cutler and Virkar [4]. The average residual stress in the matrix given by Taya et al. [7] is only a part of those presented in this paper.

\section{Analytical model}

Based on the composite sphere assemblage (CSA) model given by Hanshin [9], the two-phase model and effective model shown in Fig. 1 are used to determine the effective coefficient of thermal expansion $\alpha_{e}$ for particulate composites. The model is assumed to

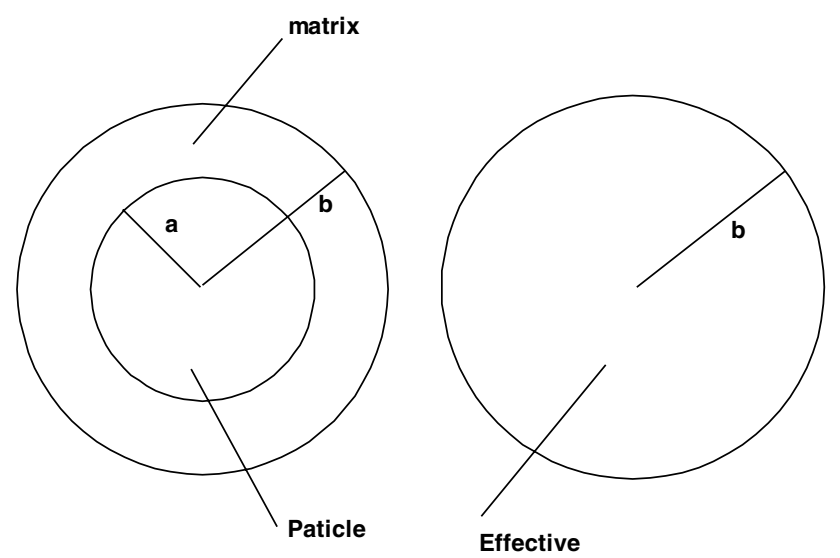

Fig. 1 The two-phase model and effective model subjected to a temperature change $\Delta T$. Introduce a spherical coordinate system with the origin at the center of the composite sphere. The general form of the thermo-elastic solutions appropriate to the two separate regions of Fig. 1a are given by [10],

$$
\begin{gathered}
u_{p}=\frac{3 \alpha_{p} K_{p} r \Delta T}{3 K_{p}+4 G_{p}}+C_{1} r \\
\sigma_{r p}=\sigma_{\theta p}=3 K_{p} C_{1}-\frac{12 \alpha_{p} K_{p} G_{p} \Delta T}{3 K_{p}+4 G_{p}} \quad 0 \leq r \leq a \\
u_{m}=\frac{3 \alpha_{m} K_{m} \Delta T}{3 K_{m}+4 G_{m}} \frac{\left(r^{3}-a^{3}\right)}{r^{2}}+C_{3} r+\frac{C_{4}}{r^{2}} \\
\sigma_{r m} \\
=-\frac{12 \alpha_{m} K_{m} G_{m} \Delta T}{3 K_{m}+4 G_{m}}\left(1-\frac{a^{3}}{r^{3}}\right)+3 K_{m} C_{3}-4 G_{m} \frac{C_{4}}{r^{3}}
\end{gathered}
$$

$\sigma_{\theta m}$

$$
\begin{aligned}
= & \frac{6 \alpha_{m} K_{m} G_{m} \Delta T}{3 K_{m}+4 G_{m}}\left(1-\frac{a^{3}}{r^{3}}\right)+3 K_{m} C_{3}+4 G_{m} \frac{C_{4}}{r^{3}} \\
& -\frac{18 \alpha_{m} K_{m} G_{m} \Delta T}{3 K_{m}+4 G_{m}} \quad a \leq r \leq b
\end{aligned}
$$

where $K$ and $G$ are bulk modulus and shear modulus, $C_{1}, C_{3}$ and $C_{4}$ are constants to be determined from the continuity conditions at the interface $r=a$ and the boundary condition at $r=b$ :

$u_{p}=u_{m}, \quad \sigma_{r p}=\sigma_{r m}$, at $r=a$

$\sigma_{r m}=0$, at $r=b$

The solution for Fig. $1 b$ is

$\begin{gathered}u_{e}=\frac{3 \alpha_{e} K_{e} r \Delta T}{3 K_{e}+4 G_{e}}+C_{5} r \\ \sigma_{r e}=\sigma_{\theta e}=3 K_{e} C_{5}-\frac{12 \alpha_{e} K_{e} G_{e} \Delta T}{3 K_{e}+4 G_{e}}\end{gathered} \quad 0 \leq r \leq b$

where $K_{e}$ and $G_{e}$ are the effective bulk modulus and shear modulus of the particle composite as given [9] and [11], $C_{5}$ is constant to be determined from the boundary condition at $r=b$,

$\sigma_{r e}=0, a t r=b$

The effective thermal expansion coefficient $\alpha_{e}$ can be derived from equality condition $u_{m}=u_{e}$ at $r=b$ for Fig. 1a and b.

$\alpha_{e}=\frac{3 K_{p} K_{m}\left(\alpha_{p} f_{p}+\alpha_{m} f_{m}\right)+4 G_{m}\left(\alpha_{p} K_{p} f_{p}+\alpha_{m} K_{m} f_{m}\right)}{3 K_{p} K_{m}+4 G_{m}\left(K_{p} f_{p}+K_{m} f_{m}\right)}$

where $f_{m}=1-f_{p}$ is the volume fraction of the matrix. 


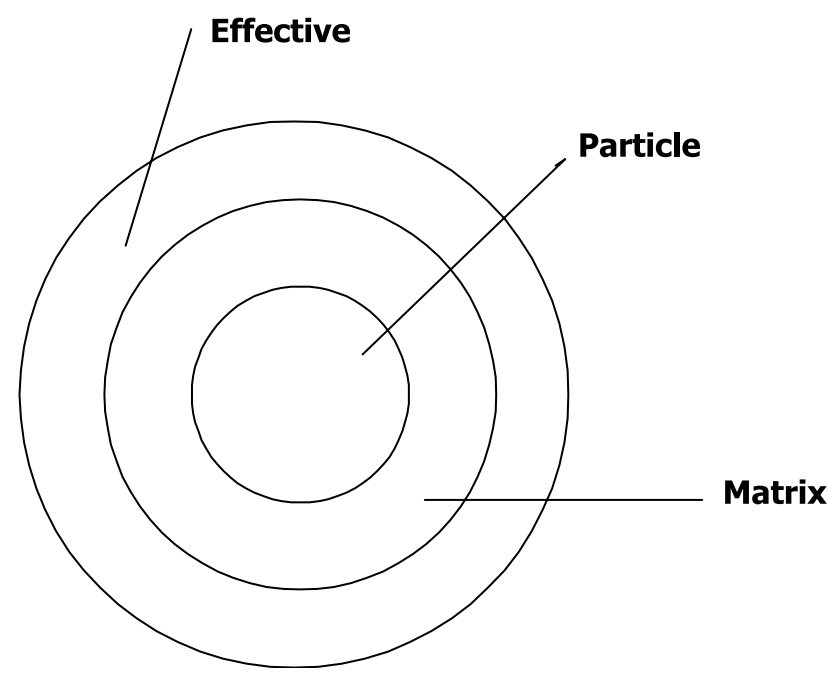

Fig. 2 The three-phase model

The residual thermal stresses will be obtained here by using the three-phase model shown in Fig. 2. The model consists of the single composite sphere embedded in the infinite homogeneous medium of the effective properties. The solutions of the particle and the matrix are given in Eqs. (3) and (4). The solution of the effective medium is

$$
\begin{gathered}
u_{e}=\frac{3 \alpha_{e} K_{e} \Delta T\left(r^{3}-b^{3}\right)}{3 K_{e}+4 G_{e}}+C_{5} r+\frac{C_{6}}{r^{2}} \\
\sigma_{r e}=-\frac{12 \alpha_{e} K_{e} G_{e} \Delta T}{3 K_{e}+4 G_{e}}\left(1-\frac{b^{3}}{r^{3}}\right)+3 K_{e} C_{5}-4 G_{e} \frac{C_{6}}{r^{3}} \quad b \leq r \leq \infty \\
\sigma_{\theta e}=\frac{6 \alpha_{e} K_{e} G_{e} \Delta T}{3 K_{e}+4 G_{e}}\left(1-\frac{b^{3}}{r^{3}}\right)+3 K_{e} C_{5}+4 G_{e} \frac{C_{6}}{r^{3}}-\frac{18 \alpha_{e} K_{e} G_{e} \Delta T}{3 K_{e}+4 G_{e}}
\end{gathered}
$$

The constants $C_{1}, C_{3}, C_{4}, C_{5}$ and $C_{6}$ in Eqs. (3),(4) and (7) can determined from the continuity conditions at the interface $r=a$ and $r=b$ and the boundary condition at $r \rightarrow \infty$ :

$u_{p}=u_{m}, \quad \sigma_{r p}=\sigma_{r m}, \quad$ at $r=a$

$u_{m}=u_{e}, \quad \sigma_{r m}=\sigma_{r e}, \quad$ at $r=b$

$\sigma_{r e}=0, \quad$ at $r \rightarrow \infty$

After some length mathematics, we get the stresses in the particle and the matrix

$\sigma_{r p}=\sigma_{\theta p}=-p \quad r \leq a$

$\begin{aligned} \sigma_{r m} & =\left(f_{p}-\frac{a^{3}}{r^{3}}\right) \frac{p}{f_{m}} \leq r \leq b \\ \sigma_{\theta m} & =\left(f_{p}+\frac{a^{3}}{2 r^{3}}\right) \frac{p}{f_{m}} \leq r\end{aligned}$

where

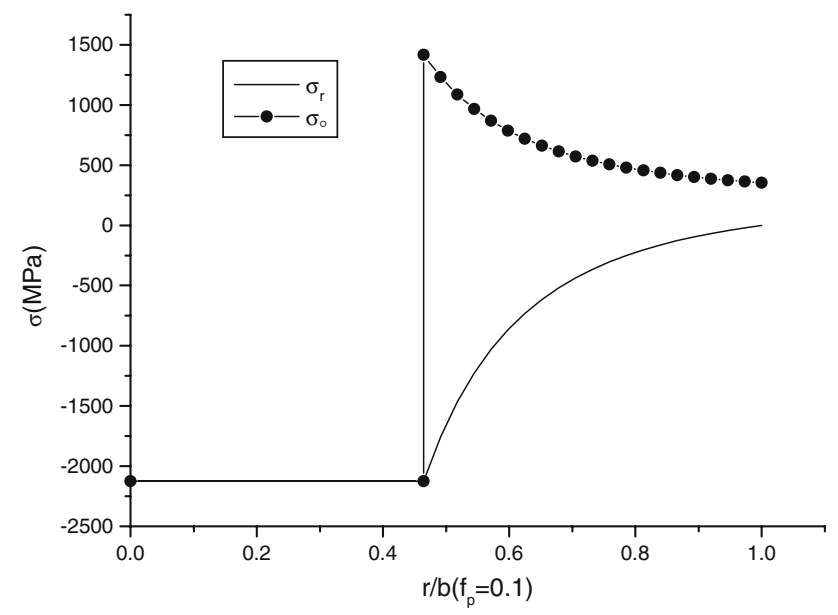

Fig. 3 The residual stresses distribution of the $\mathrm{SiC} / \mathrm{Al}_{2} \mathrm{O}_{3}$ composite

$p=\frac{12 f_{m} K_{p} K_{m} G_{m}\left(\alpha_{p}-\alpha_{m}\right) \Delta T}{3 K_{p} K_{m}+4 f_{p} K_{p} G_{p}+4 f_{m} K_{m} G_{m}}$

In the case of $f_{p}=0$ and $f_{m}=1$, the Eq. (16) reduces the (1). Therefore the Eq. (1) is a lower-order approximation to the stress in composite with a finite volume fraction of particle. Taya et al. [7] presented the formulas for calculating the average residual stresses in the particle and the matrix by using the modified Eshelby's model [8]

$\langle\sigma\rangle_{p}=-p, \quad\langle\sigma\rangle_{m}=\left(f_{p} / f_{m}\right) p$

It is clear that the average residual stress in particle is agree with Eq. (8), the stress in matrix is incomplete and only the uniform part of the stress given by Eq. (9) which is independent on the radius $r$.

\section{Numerical examples}

The Fig. 3 shows the numerical results for the SiCparticulate $\mathrm{Al}_{2} \mathrm{O}_{3}$ matrix composite. The thermal expansion coefficient, elastic modulus and Poisson's ratio of the ceramics $\mathrm{SiC}$ and $\mathrm{Al}_{2} \mathrm{O}_{3}$ are given in Table $1 . \Delta T$ is $-1630{ }^{\circ} \mathrm{C}$. The stresses $\sigma_{r}$ and $\sigma_{\theta}$ in the particulate are compression stresses. The radial stress

Table 1 Material properties

\begin{tabular}{llll}
\hline & $\mathrm{SiC}$ & $\mathrm{TiB}_{2}$ & $\mathrm{Al}_{2} \mathrm{O}_{3}$ \\
\hline$\alpha\left(\mathrm{x} 10^{-6}{ }^{\circ} \mathrm{C}\right)$ & 4.0 & 4.6 & 8.6 \\
$\mathrm{E}(\mathrm{GPa})$ & 410 & 531 & 380 \\
$v$ & 0.19 & 0.28 & 0.26 \\
\hline
\end{tabular}


Fig. 4 The residual stresses distribution of the $\mathrm{TiB}_{2} / \mathrm{SiC}$ composite

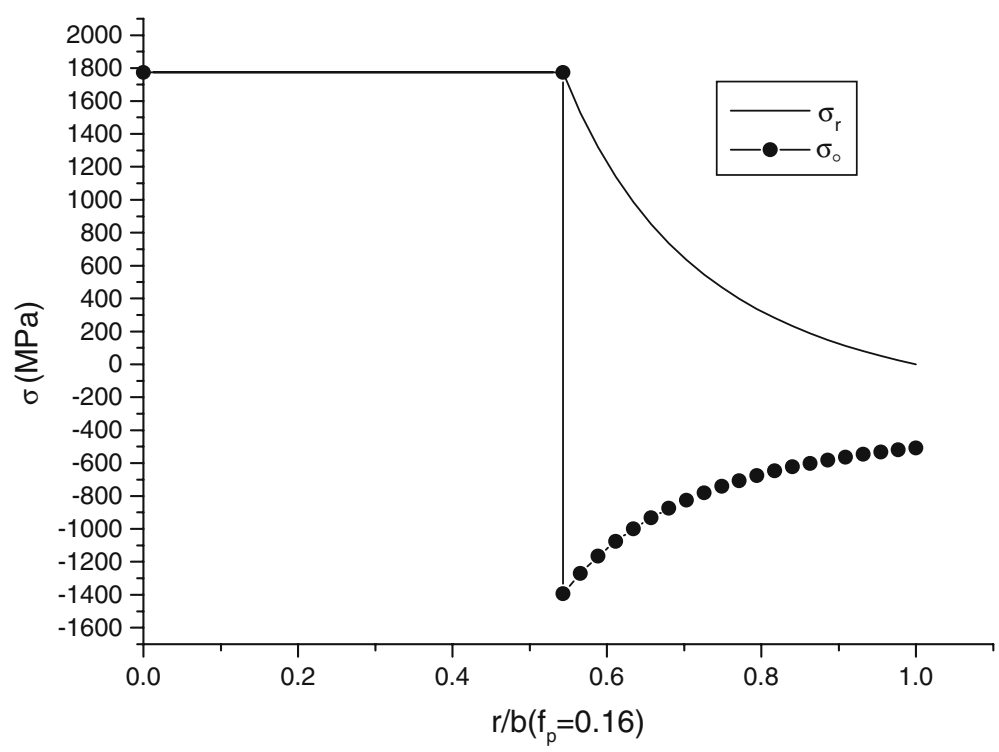

$\sigma_{r}$ in the matrix is compression stress and increases as $\mathrm{r}$ increases. But the tangential stress $\sigma_{\theta}$ in the matrix is tensile and decreases with the increase $r$.

Taya et al. [7] presented the measured increase in fracture resistance of a $\mathrm{TiB}_{2}$-particulate-reinforced SiC-matrix composite $\Delta K_{R}=2.3 \mathrm{Mpa} \mathrm{m}^{1 / 2}$. The increase in fracture resistance $\Delta K_{R}$ predicted by using Eqs.(2) and (11) is $1.56 \mathrm{MPa} \mathrm{m}^{1 / 2}$. Figure 4 shows the residual stresses distribution of the $\mathrm{TiB}_{2} / \mathrm{SiC}$ composite. It can be seen that the $\sigma_{\theta}$ and $\sigma_{r}$ in the particulate are tensile stresses. The radial stress $\sigma_{r}$ in the matrix is tensile stress and decreases as $r$ increases. The stress $\sigma_{\theta}$ in the matrix is compression stress and increases as $r$ increases. The fracture toughness, $K_{I C}$, of a particulate due to periodic residual stress field given by Eq. (9) is [5]

$K_{I C}=K_{I 0}+\sqrt{\frac{2}{\pi}} \int_{a}^{a+d} \frac{\sigma_{\theta m}}{\sqrt{a+d-x}} \mathrm{~d} x$

We get the toughness increase $\Delta K_{R}=1.74 \mathrm{MPa} \mathrm{m}^{1 / 2}$ from Eqs. (9) and (12). It is more closer the measured one.

\section{Summary}

The accurate formula for calculating the thermal residual stress field in particle-reinforced composites was presented. The increase in fracture toughness of the composite over the unreinforced matrix predicted by this formula in better agreement with the experiment [7].

\section{References}

1. Selsing G (1961) J Am Ceram Soc 44:491

2. Wei GC, Becher PF (1984) J Am Ceram Soc 67:571

3. Virkar AV, Johnson DL (1977) J Am Ceram Soc 60:514

4. Cutler RA, Virkar AV (1985) J Mater Soc 20:3557

5. Evans AG, Houer AH, Porter DL (1977) The Fracture Toughness of Ceramics, Proc. Int. Conf. Fract. 4th., p 529

6. Tada H, Paris PC, Irwin GR (1973) The Stress Analysis of Cracks Handbook; Del. Research Corp., Hellertown, PA

7. Taya M, Hayashi S, Kobayashi AS, Yoon HS (1990) J Am Ceram Soc 73:1382

8. Mori T, Tanakas K (1973) Acta Metall 21:571

9. Hanshin Z (1962) J Appl Mech 29:143

10. Timosheko S, Goodier JW (1951) Theory of elasticity, 2nd edn. McGraw Hill, New York

11. Christensen RM, Lo KH (1979) J Mech Phys Solids 27:315 